\title{
Airborne EM skin depths
}

\author{
David Beamish
}

British Geological Survey, Keyworth, Nottingham, NG12 5GG, UK

Geophysical Prospecting, 2004, 52, 439-449.

DOI: $10.1111 / \mathrm{j} .1365-2478.2004 .00428 . x$

\section{Corresponding author:}

David Beamish

British Geological Survey, Keyworth, Nottingham, NG12 5GG, UK Email: D.Beamish@bgs.ac.uk.

Tel: 01159363432

Fax: 01159363261

Keywords: Electromagnetic, airborne, frequency domain, skin depth, magnetic dipole 


\section{ABSTRACT}

Skin-depth is an electromagnetic (EM) scale length that provides a measure of the degree of attenuation experienced by a particular frequency of an EM system. As has been discussed in the literature, skin-depth is not a complete measure of the depth of investigation, but the two may be related. Frequency domain airborne EM systems employ pairs of transmitter and receiver coils that use a frequency range from several hundred hertz to over 100 kilohertz. For elevated dipoles, both geometrical and frequency dependent attenuation of the induced fields must be considered. For airborne EM systems it is possible to define a skin depth based only on the electric field induced by the transmitter. A vertical decay scale length, here defined from the at-surface position of maximum electric field, enables the same skin depth estimate to be obtained for both cases of vertical and horizontal dipolar excitation. Such dipolar skin depths associated with towed bird and fixed wing airborne systems are studied in relation to frequency, conductivity and sensor elevation. Dipolar skin depths are found to be much smaller than their plane wave counterparts except at high frequency $(>50 \mathrm{kHz})$ and in combination with high conductivity. For the majority of airborne systems the influence of altitude on skin depth is highly significant. Dipolar skin depths increase with increasing sensor elevation. Low frequencies display the greatest sensitivity. At low elevation $(<40 \mathrm{~m})$, geometrical attenuation dominates the behaviour of the skin depth. The study indicates that typical low altitude airborne surveys provide vertically compact assessments of subsurface conductivity, well suited to near surface, environmental applications.

\section{INTRODUCTION}

Frequency-domain airborne electromagnetic (AEM) systems comprise both towedbird helicopter configurations (HEM systems) and fixed-wing (wing-tip sensor) configurations. Typically, a HEM survey system operates the towed sensor bird about $30 \mathrm{~m}$ above ground level while fixed-wing systems (with larger dipole moments) may be flown much higher while maintaining adequate signal/noise. Broadly, there is an operational envelope of sensor elevations, of between $20 \mathrm{~m}$ and $100 \mathrm{~m}$, in which the 
theory of EM induction by elevated magnetic dipoles must be applied. The need to consider both high and low level surveying in populated areas, due to regulatory restrictions, has been discussed by Lee et al. (2001).

EM induction by elevated magnetic dipoles is governed both by the frequency/bandwidth of the system and the geometrical attributes of the system such as the source-receiver separation. The underlying source-receiver orientation and coupling issue has been discussed by a number of authors (Fraser, 1979; Sengpiel, 1988; Liu and Becker, 1990). If we restrict our attention to the transmitter only, then there exist only two limiting cases of dipole excitation. These are a vertically orientated coil that provides a horizontal magnetic dipole (HMD) transmitter and a horizontally orientated coil providing a vertical magnetic dipole (VMD) transmitter.

Modern towed birds provide a bandwidth from several hundred Hertz to over $100 \mathrm{kHz}$ using up to 6 pairs of transmitting and receiving coils. Coil separations are usually between 5 and $8 \mathrm{~m}$. The only, fully operational, fixed-wing AEM system is that operated by the Geological Survey of Finland (Poikonen et al. 1998). The coils are wing-tip mounted (separation of $21.4 \mathrm{~m}$ ) and are vertical coplanar. Currently, two pairs of coils provide information at two frequencies ( 3 and $14 \mathrm{kHz}$ ). The purpose of this paper is to provide a systematic study of skin depths for the typical AEM system parameters (towed-bird and fixed-wing) discussed above.

The electromagnetic skin depths considered here should not be confused with depth of penetration (or investigation). Although the two may be related, depth of investigation depends on a range of parameters that include transmitter moment together with measurement and 'geological' noise levels (Spies, 1989). In the case of AEM methods, depth of penetration was defined (Paterson, 1971; Peltoniemi, 1998) as the maximum depth from which a given conductive body gives a recognisable anomaly. In the present study only the fundamental attenuation of the AEM source fields, as described by the skin depth parameter, are considered. The attenuation has both geometrical and frequency dependent components. 
When considering AEM skin depths, AEM footprints form an associated parameter. A footprint is defined as an electromagnetic scale length at the surface of the half space. Following previous estimates based on perfectly conducting media (Kovacs et al. 1995; Liu and Becker, 1990), Beamish (2003) presented a study of AEM footprints using finitely conducting half spaces. Footprint scales increase with increasing elevation of the source. It was also noted that the at-surface footprint forms the surface expression of a volumetric skin depth in the lower half space. The maximum induced electric fields are produced at the surface and are largely geometrically imposed by the orientation and height of the transmitter. Within the earth, these atsurface fields attenuate and form a volumetric footprint that defines the principal spatial sensitivity zone of the measurement.

Sensitivity functions for geometrical, non planar source fields have been discussed for the general frequency domain EM case by McGillivray et al. (1994) and for the specific case of airborne VMD measurements by Tølbøll and Christensen (2002). These authors show that, mathematically, a magnetic field sensitivity function is given as the dot product of the electric field strength from the source and the electric field strength from the receiver assuming it acts as a source. As noted by Tølbøll and Christensen (2002), the sensitivity function is always a maximum at the surface and even for the low frequency $(385 \mathrm{~Hz}) \mathrm{VMD}$ system considered, sensitivities were low below a depth of $50 \mathrm{~m}$.

In order to illustrate the study conducted here, typical transmitter volumetric skin depths are shown in Figure 1. Figure 1a shows the induced electric field for a VMD source and Figure 1b shows the equivalent distribution for an HMD source (polarised in the x-direction). In both cases the transmitters are $90 \mathrm{~m}$ above the surface (i.e. above the origin) of a $20 \mathrm{mS} / \mathrm{m}(50 \mathrm{ohm} . \mathrm{m})$ half space and the frequency is $10 \mathrm{kHz}$. The colour contoured region defines a volume in which the modulus of the total induced electric field has decayed to 1/e of the maximum electric field (located on the surface of the half space). The SE quadrant of the region has been made transparent in order to view the interior. It should be noted that a $\mathrm{x} 4$ degree of vertical exaggeration has been applied. As discussed by Beamish (2003), the VMD provides a radially symmetric current distribution with a very localised minimum directly beneath the transmitter. The locus of the maximum electric field is a circle as indicated by colour 
and the letter $\mathrm{M}$ in Figure 1a. In the case of a HMD, the well known asymmetric (bilaterally symmetric) distribution is shown, elongate perpendicular to the direction of polarisation. The maximum electric field lies directly beneath the transmitter.

Beamish (2003) studied the at-surface skin distances (footprints) as a function of transmitter orientation (HMD and VMD), frequency, half space conductivity and altitude. It was found that the footprint scale had a primary dependence on altitude and a secondary dependence on frequency and conductivity. The purpose of the present study is to provide estimates of the corresponding behaviour of skin depths within the half space. The skin depths are shown in Figure 1 by the arrowed lines descending from the positions of maximum at-surface fields. In the case of the VMD (Fig. 1a), the skin depth occurs at a greater radial distance than the position of maximum field. In the case of the HMD (Fig. 1b), the skin depth behaviour is simpler and is located directly beneath the position of maximum field at the origin. In both cases the skin depth occurs at $29 \mathrm{~m}$ for the parameters used.

The above discussion has considered only the electric field distribution induced by the transmitter. Such skin depths are here referred to as dipolar skin depths. It is also possible to define other forms of skin depth in relation to non-planar source fields. Non-planar, or local source, skin depths were investigated by Reid and Macnae (1999) for large-scale geophysical systems. Reid and Macnae (1999) compared local source with plane wave skin depths. The authors defined a 'local source skin depth' that included the horizontal source-receiver separation, in addition to the source geometry (i.e. a VMD or HMD), the frequency, and the conductivity of the half space and the elevation of the source-receiver pair. Thus the skin depth, within the local source definition, is determined directly beneath the receiver in terms of the 'local' electric field.

The study conducted here describes the numerical methods used to calculate induced electric fields from elevated dipolar sources. Definitions of dipolar (transmitter only) skin depths are reviewed and it is demonstrated that a dipolar skin depth can be defined that is independent of dipole orientation. This allows a single skin depth to be estimated for all existing AEM systems. Such source-independent, dipolar skin depths are calculated as a function of elevation, frequency and half-space conductivity. The 
behaviour is studied across an altitude range from $20 \mathrm{~m}$ to $100 \mathrm{~m}$ thus allowing for both towed-bird and fixed-wing systems. The characteristic frequencies used here $(500 \mathrm{~Hz}, 3 \mathrm{kHz}, 14 \mathrm{kHz}, 50 \mathrm{kHz}$ and $200 \mathrm{kHz}$ ) include the two frequencies incorporated in an existing fixed-wing survey system described by Poikonen et al. (1998).

The dipolar skin depths are compared with their plane wave counterparts (these are independent of altitude). In keeping with previous results (Reid and Macnae, 1999), dipolar skin depths are much smaller than their corresponding plane wave values except at high frequencies ( $>50 \mathrm{kHz})$ and in combination with very high conductivity. Dipolar skin depths are also compared with equivalent local source skin depths calculated at typical AEM separations $(7 \mathrm{~m}$ and $21 \mathrm{~m})$. For a fixed altitude transmitter, induced electric fields remain the same provided the frequency-conductivity product remains constant. AEM skin depth behaviour with elevation is therefore summarised as a function of this product. By combining the results on vertical dipolar skin depths with existing information on at-surface skin distance footprints, it is also possible to summarise the volumetric attenuation behaviour of the current distributions induced by AEM systems.

\section{METHODOLOGY}

Skin depth is an electromagnetic scale length that provides a measure of the degree of attenuation experienced by a particular frequency of an EM system. For consistency, uniform half space conductivities are usually employed. The skin depth may be defined as the depth at which the amplitude of the plane wave electric field falls to 1/e (0.368) of its surface value (Spies, 1989). The frequency domain plane wave skin depth is defined as:

$\delta=\sqrt{2 /(\sigma \mu \omega)}$

where $\sigma$ is the half space conductivity, $\mu$ is the magnetic permeability and $\omega$ is angular frequency. It is assumed that conductivity and magnetic permeability are frequency independent and that displacement currents can be ignored. 
The skin depth provides a measure of frequency dependent electromagnetic attenuation for plane wave fields. For elevated dipoles, geometrical attenuation must also be considered. In free space conditions the primary magnetic field from a small loop (a point dipole) decays with distance (r) as $r^{3}$. This spatial decay is referred to as geometrical attenuation. The introduction of a finitely conducting body results in secondary field components in addition to the primary field. Both primary and secondary fields exhibit geometrical attenuation that varies from $r^{3}$ to $r^{6}$ depending on the size and geometry of the conductive body (Peltoniemi, 1998).

The electromagnetic solution for an elevated magnetic dipole above a homogenous earth was developed by Wait (1955). The model was extended to the 2-layer case by Frischknecht (1967). Anderson (1979) and Siemon (2001) discuss subsequent generalisation to a layered half space. The frequency domain electromagnetic vector potentials can be used to obtain the quasi static electric and magnetic fields within the half-space. Here, although the frequency analysis extends to $200 \mathrm{kHz}$, quasi-static (low frequency) behaviour is assumed.

Accurate techniques for the numerical integration of the expressions have been established (Frischknecht, 1967; Anderson, 1979, Christensen, 1990) and the calculation of induced electric fields follows that of Beamish (2003). Following Ward (1967), the expressions for the total (free space primary and secondary) electric field within a half-space $(z \geq 0)$ in the plane $y=0$ are given by:

$E_{y}=-\frac{i \omega \mu M}{2 \pi} \int_{0}^{\infty} \frac{\lambda^{2}}{\lambda+m} e^{-\lambda h} e^{-m z} J_{1}(\lambda x) d \lambda$

for a VMD in the $+\mathrm{z}$ direction, and

$$
\begin{aligned}
\mathrm{E}_{y}=-\frac{i \omega \mu \mathrm{M}}{2 \pi}\left\{\frac{1}{x} \int_{0}^{\infty} \frac{\lambda^{2}}{\lambda+\mathrm{m}} \mathrm{e}^{-\lambda \mathrm{h}} \mathrm{e}^{-\mathrm{mz}} \mathrm{J}_{1}(\lambda x) \mathrm{d} \lambda\right. \\
\left.+\int_{0}^{\infty} \frac{\lambda^{2}}{\lambda+\mathrm{m}} \mathrm{e}^{-\lambda \mathrm{h}} \mathrm{e}^{-\mathrm{mz}} \mathrm{J}_{0}(\lambda x) \mathrm{d} \lambda\right\}
\end{aligned}
$$


for a HMD in the $+x$ direction, and where $\mathrm{m}=\sqrt{\left(\lambda^{2}-i \omega \mu \sigma\right)}$, $\sigma$ being the conductivity of the half-space and $\lambda$ is wavenumber. In these expressions the dipole is at a height $\mathrm{h}$ above the surface of the half-space, $\mathrm{z}$ is the depth below the surface, $\mathrm{M}$ is the dipole moment and $\mathrm{J}_{0}(\lambda x)$ and $\mathrm{J}_{1}(\lambda x)$ are Bessel functions of the first kind of orders 0 and 1 , respectively.

The electric fields are calculated on a grid within the half space (e.g. Reid and Macnae, 1999; Beamish, 2003). In the present study vertical and horizontal grid intervals of $<0.5 \mathrm{~m}$ were used. The basic calculation of skin-depth requires the determination of the depth at which the amplitude of the field has reduced to 1/e of the maximum surface value. As discussed below, the induced field is a complex vector quantity comprising in-phase and in-quadrature components. Skin depth calculations have been made using the modulus of the total horizontal electric field.

\section{THE INDUCED ELECTRIC FIELD}

The complex (phase) behaviour of the current system induced by the transmitter is discussed first. The phase behaviour considered, that of the total induced electric field (arising solely from the transmitter), should not be confused with the phase behaviour of the magnetic coupling ratios between transmitter and receiver. Figure 2 shows the in-phase $(\mathrm{P})$ and in-quadrature $(\mathrm{Q})$ components of the induced electric field due to a HMD (polarised in the x-direction) transmitter (Fig. 2a,b) and a VMD transmitter (Fig. 2c,d) above a $10 \mathrm{mS} / \mathrm{m}$ (100 ohm.m) half-space. Since the distributions are symmetrical about the origin (along the $\mathrm{x}$-axis) only the cross sections between $\mathrm{x}=0$ and $x=50 \mathrm{~m}$ are shown. The frequency used is $3 \mathrm{kHz}$ and the dipoles are located 30 $\mathrm{m}$ above the origin. The components of the horizontal electric field have been normalised to unity in each plot. For both HMD and VMD sources, the Q components are larger than the $\mathrm{P}$ components by a factor of about 10. In the case of the HMD (Fig. $2 \mathrm{a}, \mathrm{b})$ the maximum amplitude occurs in the $\mathrm{Q}$ component at the surface and is located directly below the transmitter $(\mathrm{x}=0 \mathrm{~m})$. This value is used to normalise both the $\mathrm{P}$ and Q components of the HMD distributions. The P component values are shown multiplied by a factor of 10 . The maximum amplitude in the case of the VMD occurs 
in the Q component at the surface at an offset of $21 \mathrm{~m}$ from the origin. This value is used to normalise both the $\mathrm{P}$ and $\mathrm{Q}$ components of the VMD distributions. Once again, the $\mathrm{P}$ component values are shown multiplied by a factor of 10 . In each of the plots, amplitudes greater than 0.5 are shaded to emphasise the zones of maximum induced field. The weaker P component distributions both possess maxima below the surface and form relatively large diffuse zones. The Q component distributions form the main decay behaviour of the induced electric field within the half space, for the parameters used. Both the HMD and VMD Q components possess maximum amplitudes at the surface of the half-space. This general form of the behaviour of the $\mathrm{P}$ and $\mathrm{Q}$ component maxima is repeated at all frequencies (e.g. Tølbøll and Christensen, 2002).

The induced current distributions remain the same provided the frequencyconductivity product remains constant. It is possible to summarise the phase behaviour of the induced field as a function of this product. Three ranges of the frequency-conductivity product have been considered. A frequency-conductivity product of $0.1 \mathrm{~Hz} . \mathrm{S} / \mathrm{m}$ corresponds to the low frequency and/or low conductivity range of AEM systems (e.g. $100 \mathrm{~Hz}$ and $0.001 \mathrm{~S} / \mathrm{m}$ ). At this limit, the induced current system is effectively in-quadrature (a phase variation of $90^{\circ}$ to $89.7^{\circ}$ over the height range from $20 \mathrm{~m}$ to $100 \mathrm{~m}$ ). A frequency-conductivity product of $100 \mathrm{~Hz} . \mathrm{S} / \mathrm{m}$ corresponds to the mid frequency and/or mid conductivity range of AEM systems (e.g. $1000 \mathrm{~Hz}$ and $0.100 \mathrm{~S} / \mathrm{m}$ ). For this product, the phase of the current system is predominantly in quadrature (a phase variation of $85^{\circ}$ to $69^{\circ}$ over the height range from $20 \mathrm{~m}$ to $100 \mathrm{~m}$ ). A frequency-conductivity product of $10^{5} \mathrm{~Hz} . \mathrm{S} / \mathrm{m}$ corresponds to the high frequency and/or high conductivity range of AEM systems (e.g.10 5 Hz and 1 $\mathrm{S} / \mathrm{m})$. For this product, the phase of the current system approaches, but does not reach $45^{\circ}$ (a phase variation of $51^{\circ}$ to $46^{\circ}$ is observed across the height range from $20 \mathrm{~m}$ to $100 \mathrm{~m})$.

It is apparent that the phase of the current system of dipolar AEM systems is limited to between $90^{\circ}$ and $45^{\circ}$. The lower limit is produced as the half-space approaches a perfect conductor. With reference to Figure 2, the maximum induced fields are observed at the surface of the half space over all frequencies and for both transmitter orientations. 


\section{DIPOLAR SKIN DEPTHS}

The dipolar skin depths discussed here are an electrical scale length that depends only on the orientation of the transmitter, its elevation and the conductivity of the half space. As has already been stated, the maximum induced electric field occurs at the surface of the half-space. For an HMD source, the maximum occurs directly below the transmitter. For a VMD source, the maximum occurs at an offset that is primarily determined by the elevation of the source. Simple formulae for the estimation of the location of the maximum current are given by Beamish (2003). As an example, Figure 3 shows the total electric field in the x-z plane due to an HMD source (polarised in the $\mathrm{x}$ direction) and a VMD source above a $10 \mathrm{mS} / \mathrm{m}$ half-space. The sources are $30 \mathrm{~m}$ above the origin $(\mathrm{x}=0 \mathrm{~m})$. The field is normalised to unity in each case and a contour interval of 0.05 is used. The skin depth contour (a decay of $1 / \mathrm{e}=0.368$ ) is shown by the heavy contour and the region with values $>1 / \mathrm{e}$ is shaded.

In the case of the HMD (Fig. 3a) the skin depth estimate may be simply defined directly below the origin as shown by the arrow. For this example the skin depth estimate is $19 \mathrm{~m}$. An identical estimate is obtained using an HMD source polarised in the $y$ direction. In the case of the VMD (Fig. 3b), the skin depth estimate must be found by determining the maximum depth of the 1/e contour across the section. As shown in Figure 3b, this depth occurs at an increased offset from the surface position of maximum current $(21 \mathrm{~m})$. The estimate of skin depth obtained in this way is again $19 \mathrm{~m}$. In both cases the estimate of skin depth is obtained using a numerical grid in the $\mathrm{x}-\mathrm{z}$ plane that, in this case, has a resolution of $0.5 \mathrm{~m}$.

The definition of skin depth used above has the advantage that it is independent of source orientation. A single skin depth, as defined above, can be used to investigate the behaviour of both types of dipole transmitter. 


\section{DIPOLAR SKIN DEPTHS AS A FUNCTION OF ELEVATION, FREQUENCY AND CONDUCTIVITY}

The behaviour of dipolar skin depths is first examined as a function of frequency only. This allows a direct comparison with plane-wave skin depths in a half space. The parameters follow from the previous examples and a $10 \mathrm{mS} / \mathrm{m}$ half-space is used with a dipole elevation increased to $40 \mathrm{~m}$. Five frequencies between 0.5 and $200 \mathrm{kHz}$ are examined in this first example.

Figure 4a shows the normalised plane wave E-field decay curves within a $10 \mathrm{mS} / \mathrm{m}$ half space for the range of frequencies considered. The curves necessarily display exponential decay from the normalised surface value of unity. The dash line with an E-field amplitude of 0.368 locates the skin depth for each frequency displayed (above a cut-off depth of $60 \mathrm{~m}$ ). The dipolar decay curves shown in Figure $4 \mathrm{~b}$ are the numerically determined equivalent curves for a VMD source at an elevation of $40 \mathrm{~m}$. The same behaviour is observed for an HMD source. It is immediately apparent that for frequencies below $200 \mathrm{kHz}$, the E-field decay of the dipolar source undergoes a high degree of geometrical attenuation when compared to plane-wave behaviour. For this fixed elevation analysis, plane-wave behaviour (implying a long wavelength source field) is only observed as the frequency exceeds $50 \mathrm{kHz}$. Skin depths for the plane-wave analysis range from $11 \mathrm{~m}$ (at $200 \mathrm{kHz}$ ) to $225 \mathrm{~m}$ (at $0.5 \mathrm{kHz}$ ); in the case of the dipolar source, skin depths only vary between $11 \mathrm{~m}$ (at $200 \mathrm{kHz}$ ) to just over 25 $\mathrm{m}$ at $0.5 \mathrm{kHz}$.

As previously discussed, it is necessary to conduct an analysis that includes transmitter elevation to take into account different wavenumber contributions. Figure 5 shows the results obtained for two half-spaces of $10 \mathrm{mS} / \mathrm{m}$ and $100 \mathrm{mS} / \mathrm{m}$. Four frequencies are considered and the source elevation ranges from $20 \mathrm{~m}$ to $100 \mathrm{~m}$. Plane wave skin depths for the $10 \mathrm{mS} / \mathrm{m}$ half-space are $225 \mathrm{~m}(0.5 \mathrm{kHz}), 91.8 \mathrm{~m}(3 \mathrm{kHz})$,

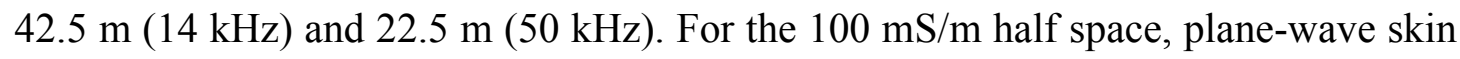
depths are $71 \mathrm{~m}(0.5 \mathrm{kHz}), 29 \mathrm{~m}(3 \mathrm{kHz}), 13.5 \mathrm{~m}(14 \mathrm{kHz})$ and $7.1 \mathrm{~m}(50 \mathrm{kHz})$.

It is evident from Figure 5 that dipolar skin depths increase with increasing elevation. The lowest frequencies and least conductive environments produce the greatest 
dependence on elevation. At high frequencies and high conductivities, the dependence on elevation is greatly reduced and plane-wave behaviour is approached.

\section{LOCAL-SOURCE SKIN-DEPTHS}

Geometrical, or local source, skin depths were investigated by Reid and Macnae (1999) for large source-receiver separation geophysical systems. The authors calculated the depth in a homogeneous half space where the amplitude of VMD and HMD source fields falls to $1 / \mathrm{e}$ of their surface value. The authors defined a 'local source' skin depth that included the horizontal source-receiver separation, in addition to the source geometry (i.e. a VMD or HMD), the frequency, the conductivity of the half space and the elevation of the source-receiver pair. Thus the skin depth, within the local source definition, is determined directly beneath the receiver in terms of the 'local' electric field. The procedure is to first calculate the surface electric field at a fixed offset (the source-receiver separation) and then to calculate the depth, vertically below the fixed offset, at which the field amplitude falls to 1/e of this value. The study by Reid and Macnae (1999) did not specifically include AEM systems and the height dependence issue.

The behaviour of local source skin depths can be described by using the induced electric field cross sections shown previously in Figure 3. The transmitter is located above the origin and local source skin depths can be determined at any prescribed offset. Here we consider the two generic AEM separations of 7 and $21 \mathrm{~m}$. In the case of the HMD (Fig. 3a), a $7 \mathrm{~m}$ local source skin depth would behave in a similar manner to that of the dipolar skin depth since the offset is located close to the position of the maximum field. A $21 \mathrm{~m}$ local source skin depth would behave differently. In the case of the VMD source (Fig. 3b), a $21 \mathrm{~m}$ local source skin depth would be similar to the dipolar behaviour since this offset is very close (coincidentally) to the location of maximum field. A $7 \mathrm{~m}$ local source skin depth would, in the case of the VMD source, behave differently. 
Reid and Macnae (1999) found that with increasing offset from the source, the local source skin depth increases to a maximum value of almost twice the plane wave skin depth and then decreases until it becomes equal to the plane wave skin depth at large offsets. Close to the transmitter (small offsets), the local source skin depth is less than the plane wave skin depth. This is the situation when considering all towed-bird and fixed wing AEM systems.

For completeness, a study of local source skin depths has been undertaken; only an example is provided here. In the case of local source skin depths it is necessary to consider both transmitter geometry and source-receiver offset. Both VMD and HMD sources and generic offsets of 7 and $21 \mathrm{~m}$ are considered. Figure 6 shows the local source skin depths, compared with the dipolar skin depth, over an elevation range from 20 to $100 \mathrm{~m}$. A half space conductivity of $10 \mathrm{mS} / \mathrm{m}$ is used and two frequencies of $500 \mathrm{~Hz}$ (Fig. 6a) and $50 \mathrm{kHz}$ (Fig. 6b) are shown. The higher frequency results are closer to plane wave (altitude independent) skin depth behaviour. Plane wave skin depths are $225 \mathrm{~m}$ at $500 \mathrm{~Hz}$ and $22.5 \mathrm{~m}$ at $50 \mathrm{kHz}$.

Except at the lowest elevations, the local source skin depths due to an HMD source exceed those due to a VMD source. This is a similar result to that obtained by Reid and Macnae (1999). The authors indicate that the maximum local source skin depth for an elevated HMD transmitter, based on the amplitude of the electric field, is greater than that for a VMD transmitter at the same height. Figure 6 indicates that for a given source geometry, differences between the $7 \mathrm{~m}$ and $21 \mathrm{~m}$ source-receiver separations are only slight. The behaviour of the local-source skin depth with elevation for the HMD source is found to be similar to that of the dipolar skin depth.

\section{SUMMARY}

As noted previously, the induced current distributions remain the same provided the frequency-conductivity product remains constant. It is possible to summarise the behaviour of the dipolar skin depth with elevation by considering a sequence of results for different frequency-conductivity products. The results obtained for five values of the frequency-conductivity product are shown in Figure 7. A frequency- 
conductivity product of $1 \mathrm{~Hz} . \mathrm{S} / \mathrm{m}$ corresponds to the low frequency and/or low conductivity range of AEM systems (e.g. $1000 \mathrm{~Hz}$ and $0.001 \mathrm{~S} / \mathrm{m}$ ). Frequencyconductivity products of $10 \mathrm{~Hz} . \mathrm{S} / \mathrm{m}, 100 \mathrm{~Hz} . \mathrm{S} / \mathrm{m}$ and $1000 \mathrm{~Hz} . \mathrm{S} / \mathrm{m}$ (e.g. $10 \mathrm{kHz}$ and $0.1 \mathrm{~S} / \mathrm{m}$ ) then progress towards the higher frequency and/or higher conductivity ranges. Finally a frequency-conductivity product of $10^{4} \mathrm{~Hz} . \mathrm{S} / \mathrm{m}$ (e.g. $100 \mathrm{kHz}$ and 0.1 $\mathrm{S} / \mathrm{m}$ ) corresponds to the high frequency and/or high conductivity range of AEM systems. Plane wave skin depths for the models are $503 \mathrm{~m}(1 \mathrm{~Hz} . \mathrm{S} / \mathrm{m}), 159 \mathrm{~m}(10$ Hz.S/m), 50.3 m (100 Hz.S/m), 15.9 m (1000 Hz.S/m) and 5.0 m (10 Hz.S/m).

The results indicate that when the frequency-conductivity product lies in the range from $<1$ to $100 \mathrm{~Hz} . \mathrm{S} / \mathrm{m}$ (i.e. low frequency and/or low conductivity) then the dipolar nature of the skin depth is highly significant. A strong dependence on elevation is observed and skin depths are significantly less than the corresponding plane wave values. As the frequency-conductivity product increases to values of $1000 \mathrm{~Hz} . \mathrm{S} / \mathrm{m}$ and above, plane-wave behaviour is increasingly observed in the behaviour of the dipolar skin depths at all elevations.

It is also possible to summarise the volumetric behaviour of the induced current distributions using the dipolar skin depths (in the vertical plane) considered in the present study and the at-surface skin distance footprints (in the horizontal plane) considered by Beamish (2003). Using the approximate formulae, for variation with elevation given in Table 1 of Beamish (2003), a long skin distance (LSD) is associated with an HMD source and an outer skin distance (OSD) is associated with a VMD source. Parameters are given for a mid-range frequency of $10 \mathrm{kHz}$ and for half space conductivities of 1 and $100 \mathrm{mS} / \mathrm{m}$.

Figure 8 shows the variation of dipolar skin depth and at-surface skin distance footprint for source elevations from 20 to $100 \mathrm{~m}$, at a frequency of $10 \mathrm{kHz}$. Results are shown for a conductivity of $100 \mathrm{mS} / \mathrm{m}$ (Fig. 8a) and for $1 \mathrm{mS} / \mathrm{m}$ (Fig. 8b). As described by Beamish (2003), horizontal footprints for the VMD source are significantly greater than their HMD counterparts. In both sets of results, vertical skin depths are less than the smallest horizontal footprint scale (lowest elevation). In the conductive environment (Fig. 8a), the insensitivity of skin depth to source elevation leads to dramatic increases in the horizontal to vertical scale of the skin volume. With 
increasing elevation, the skin volume as measured by the horizontal to vertical scale of skin distances, becomes increasingly compressed. At an elevation of $30 \mathrm{~m}$, horizontal to vertical skin distance ratios are 3.24 for the HMD and 4.51 for the VMD source. As the elevation increases to $90 \mathrm{~m}$, these ratios increase to 6.43 and 8.71, respectively. The behaviour is much less pronounced for the low conductivity results shown in Figure 8b. At an elevation of $30 \mathrm{~m}$, horizontal to vertical skin distance ratios are 2.45 for the HMD and 3.62 for the VMD source. As the elevation increases to 90 $\mathrm{m}$, these ratios increase only marginally to 2.53 and 3.74 , respectively.

\section{CONCLUSIONS}

The study conducted here has examined dipolar skin depths which form a basic attenuation scale length parameter associated with elevated magnetic dipole sources. The parameters used, elevations from $20 \mathrm{~m}$ to $100 \mathrm{~m}$ and frequencies from $0.5 \mathrm{kHz}$ to $200 \mathrm{kHz}$, encompass the ranges of existing towed bird and fixed wing airborne systems. It was demonstrated that for these systems it is possible to define a skin depth in the vertical plane, based only on the modulus of the total electric field induced in the lower half space by the transmitter. This definition, using the at-surface position of maximum electric field, enables the same value of skin depth to be associated with both vertical and horizontal dipole orientations.

The dipolar skin depths are found to be much smaller than their plane wave counterparts except at high frequency $(>50 \mathrm{kHz})$ and in combination with high conductivity. Dipolar skin depths increase with increasing sensor elevation. Lower frequencies, in combination with low conductivity, display the greatest sensitivity. Both the geometrical and frequency dependent behaviour of AEM skin depths with elevation have been summarised using a constant frequency-conductivity product. The results have also been compared with an alternative, existing definition of 'local source' skin depths. This latter definition requires both dipole orientation and transmitter-receiver separation to be specified. It was found that the local source skin depths for a HMD source using separations of $7 \mathrm{~m}$ and $21 \mathrm{~m}$ display similar behaviour to that of the dipolar skin depth. 
The vertical dipolar skin depths studied here can be combined with existing information on at-surface skin distance footprints. This allows a summary of the volumetric form of the attenuation of the fields induced by both HMD and VMD sources. Results have been presented for a mid-range frequency $(10 \mathrm{kHz})$. In a conductive environment $(100 \mathrm{mS} / \mathrm{m})$ and for a source elevation of $30 \mathrm{~m}$, horizontal to vertical skin distance ratios are 3.2 for a HMD and 4.5 for a VMD source. As the elevation increases threefold to $90 \mathrm{~m}$, these ratios increase to 6.4 and 8.7. The altitude dependence is much less pronounced in low conductivity environments.

It is clear from the horizontal to vertical skin distance ratios that AEM systems typically provide vertically compact skin volumes. In addition, maximum induced electric fields and hence sensitivities occur at the surface. Such behaviour, although inevitably limited in lateral resolution, is well suited to environmental and land quality applications. The majority of sources of environmental impact exist at the surface (e.g. various forms of mineral loading) or they may have been excavated from the surface (e.g. landfills). There is then a requirement to use appropriate geophysical survey data to both define a likely source zone (i.e. its near-surface volumetric limits) and to examine whether any lateral and vertical migration of source constituents can be detected. Vertical scales of assessment of a few tens of metres over a depth range of some 50 to $100 \mathrm{~m}$, are appropriate and can be supplied by AEM surveys.

\section{ACKNOWLEDGEMENT}

This report is published with the permission of the Executive Director, British Geological Survey (NERC).

\section{REFERENCES}

Anderson, W.L. 1979. Numerical integration of related Hankel transforms of order 0 and 1 by adaptive digital filtering. Geophysics 44, 1287-1305.

Beamish, D. 2003. Airborne EM footprints. Geophysical Prospecting 51, 49-60. 
Christensen, N.B. 1990. Optimized fast Hankel transform filters. Geophysical Prospecting 38, 545-568.

Fraser, D.C. 1979. The multicoil II airborne electromagnetic system. Geophysics 44, 1367-1394.

Frischknecht, F.C. 1967. Fields about an oscillating magnetic dipole over a two-layer earth and application to ground and airborne electromagnetic surveys. Colorado School of Mines Quarterly 62, 326pp.

Kovacs, A., Holladay, J.S. and Bergeron Jr., C.J. 1995. The footprint/altitude ratio for helicopter electromagnetic sounding of sea-ice thickness: Comparison of theoretical and field estimates. Geophysics 60, 374-380.

Lee, M.K., Peart, R.J., Jones, D.G., Beamish, D., and Vironmaki, J., 2001. Applications and challenges for high resolution airborne surveys in populated areas. EAGE $63^{\text {nd }}$ Conference, Amsterdam, Extended Abstracts, Paper IA-1.

Liu, G. and Becker, A. 1990. Two-dimensional mapping of sea-ice keels with airborne electromagnetics. Geophysics 55, 239-248.

McGillivray, P.R., Oldenburg, D.W., Ellis, R.G. and Habashy, T.M. 1994. Calculation of sensitivities for the frequency domain electromagnetic problem. Geophys. J. Int. 116, 1-4.

Paterson, N.R. 1971. Airborne electromagnetic methods as applied to the search for sulphide deposits. CIM Transactions 74, 1-10.

Peltoniemi, M., 1998. Depth of penetration of frequency-domain airborne electromagnetics in resistive terrains. Exploration Geophysics 29, 12-15. 
Poikonen, A., Sulkanen, K, Oksama, M., and Suppala, I. 1998. Novel dual frequency fixed wing airborne EM system of Geological Survey of Finland (GTK). Exploration Geophysics 29, 46-51.

Reid J.E., and Macnae, J.C. 1999. Doubling the effective skin depth with a local source. Geophysics 64, 732-738.

Sengpiel, K.-P. 1988. Approximate inversion of airborne EM data from a multilayered ground. Geophysical Prospecting 36, 446-459.

Siemon, B. 2001. Improved and new resistivity-depth profiles for helicopter electromagnetic data. Journal of Applied Geophysics 46, 65-76.

Spies, B.R. 1989. Depth of investigation in electromagnetic sounding methods. Geophysics 54, 872-888.

Tølbøll R.J. and Christensen, N.B. 2002. The sensitivity functions of airborne frequency domain methods. Proceedings of the $8^{\text {th }}$ meeting of the EEGS, Aveiro, Portugal, 539-542.

Wait, J.R. 1955. Mutual electromagnetic coupling of loops over a homogenous ground. Geophysics 20, 630-637.

Ward, S.H. 1967. Electromagnetic theory for geophysical applications. In: Mining geophysics, Vol. II, Theory (eds. D.A. Hansen, W.E. Heinrichs, R.C. Holmer, R.E. MacDougall, G.R. Rogers, J.S. Dumner and S.H. Ward), pp. 10-196. Soc. Expl. Geophys., Tulsa, OK. 


\section{FIGURE CAPTIONS}

Figure 1 Three dimensional perspective views of induced electric field skin distance volumes (the contoured region) generated by magnetic dipoles at a frequency of $10 \mathrm{kHz}, 90 \mathrm{~m}$ above a $20 \mathrm{mS} / \mathrm{m}$ half-space. (a) Vertical Magnetic Dipole (VMD) and (b) Horizontal Magnetic Dipole (HMD) polarised in the x-direction. The volume shown $(200 \times 200 \times 30 \mathrm{~m})$ has a vertical exaggeration of $\times 4$. The SE quadrant has been removed.

Figure 2 Cross sections, in the $\mathrm{x}-\mathrm{z}$ plane, of the induced electric field components P (in-phase) and Q (in-quadrature) due to (a) an Horizontal Magnetic Dipole (HMD) source polarised in the x direction, and (b) a Vertical Magnetic Dipole (VMD) source. In both cases the half space conductivity is $10 \mathrm{mS} / \mathrm{m}$, the frequency is $3 \mathrm{kHz}$ and the source is $30 \mathrm{~m}$ above the origin. The distributions have been normalised to a maximum value of unity and the in-phase values are shown multiplied by 10 . The maximum value is unity and the contour interval is 0.05 throughout. The contour interval is linear.

Figure 3 Cross sections, in the $\mathrm{x}-\mathrm{z}$ plane, of the modulus of the induced electric field due to an Horizontal Magnetic Dipole (HMD) source polarised in the $\mathrm{x}$ direction, and (b) a Vertical Magnetic Dipole (VMD) source. In both cases the half space conductivity is $10 \mathrm{mS} / \mathrm{m}$, the frequency is $3 \mathrm{kHz}$ and the source is $30 \mathrm{~m}$ above the origin. The distributions have been normalised to a maximum value of unity in each case and the contour interval is 0.05 . The heavy line contour (a value of 0.37 ) denotes a skin distance of 1/e from the maximum value of unity at the surface.

Figure 4 Comparison of the attenuation of induced electric fields, normalised to a value of unity at the surface, in the case of (a) a plane wave source and (b) a Vertical Magnetic Dipole (VMD) source, located $40 \mathrm{~m}$ above the half space. The half space conductivity is $10 \mathrm{mS} / \mathrm{m}$. Results for 5 frequencies are shown. The vertical dashed line denotes a value of $1 / \mathrm{e}(0.37)$ i.e. the skin depth. 
Figure 5 Variation of dipolar skin depth with source elevation and frequency for two half spaces (a) $10 \mathrm{mS} / \mathrm{m}$ and (b) $100 \mathrm{mS} / \mathrm{m}$. Results for 4 frequencies are shown as individual curves in each case.

Figure 6 Comparison of dipolar skin depths (solid lines) with local source skin depths (symbols) for a half space of conductivity $10 \mathrm{mS} / \mathrm{m}$ and for frequencies of (a) $500 \mathrm{~Hz}$ and (b) $50 \mathrm{kHz}$. Local source skin depths are shown for Vertical Magnetic Dipoles (VMD) and for Horizontal Magnetic Dipoles (HMD) with transmitterreceiver offsets of $7 \mathrm{~m}$ (bird) and $21 \mathrm{~m}$ (fixed wing).

Figure 7 Variation of dipolar skin depth with elevation as a function of the frequency-conductivity product $\left(f^{*} \Phi\right)$. Five curves for the conductivity-frequencyconductivity product ranging from 1 to $10000 \mathrm{~Hz} . \mathrm{S} / \mathrm{m}$ are shown.

Figure 8 Variation of dipolar vertical skin depth with horizontal skin distance parameters (footprints) for VMD and HMD sources at elevations ranging from 20 to $100 \mathrm{~m}$. The numbers to the right of each symbol denote source elevation. The frequency used is $10 \mathrm{kHz}$. (a) Results for a half space conductivity of $100 \mathrm{mS} / \mathrm{m}$ and (b) Results for a half space conductivity of $1 \mathrm{mS} / \mathrm{m}$. 


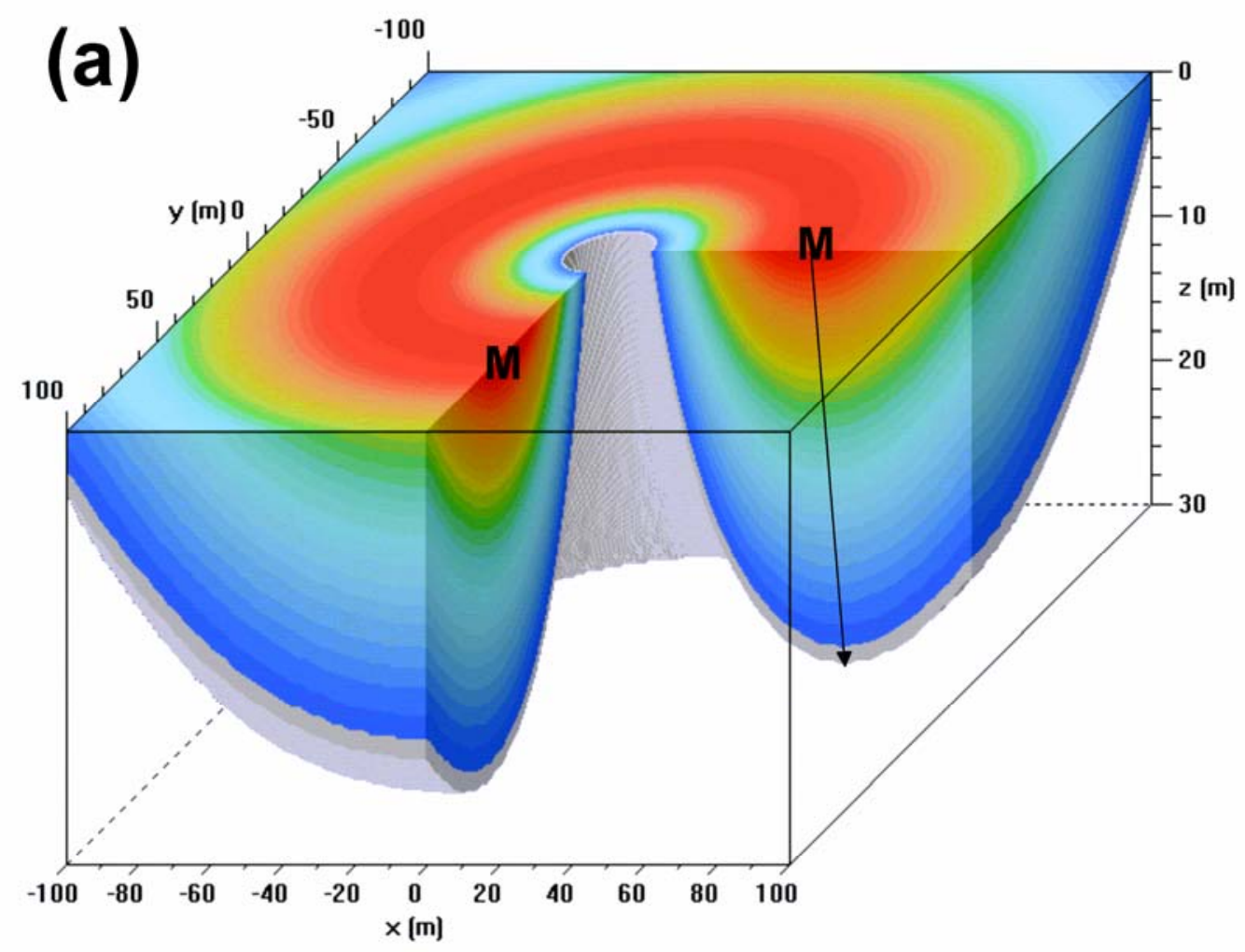

Figure 1 Three dimensional perspective views of induced electric field skin distance volumes (the contoured region) generated by magnetic dipoles at a frequency of 10 kHz, $90 \mathrm{~m}$ above a $20 \mathrm{mS} / \mathrm{m}$ half-space. (a) Vertical Magnetic Dipole (VMD) and (b) Horizontal Magnetic Dipole (HMD) polarised in the x-direction. The volume shown $(200 \times 200 \times 30 \mathrm{~m})$ has a vertical exaggeration of $\times 4$. The SE quadrant has been removed. 


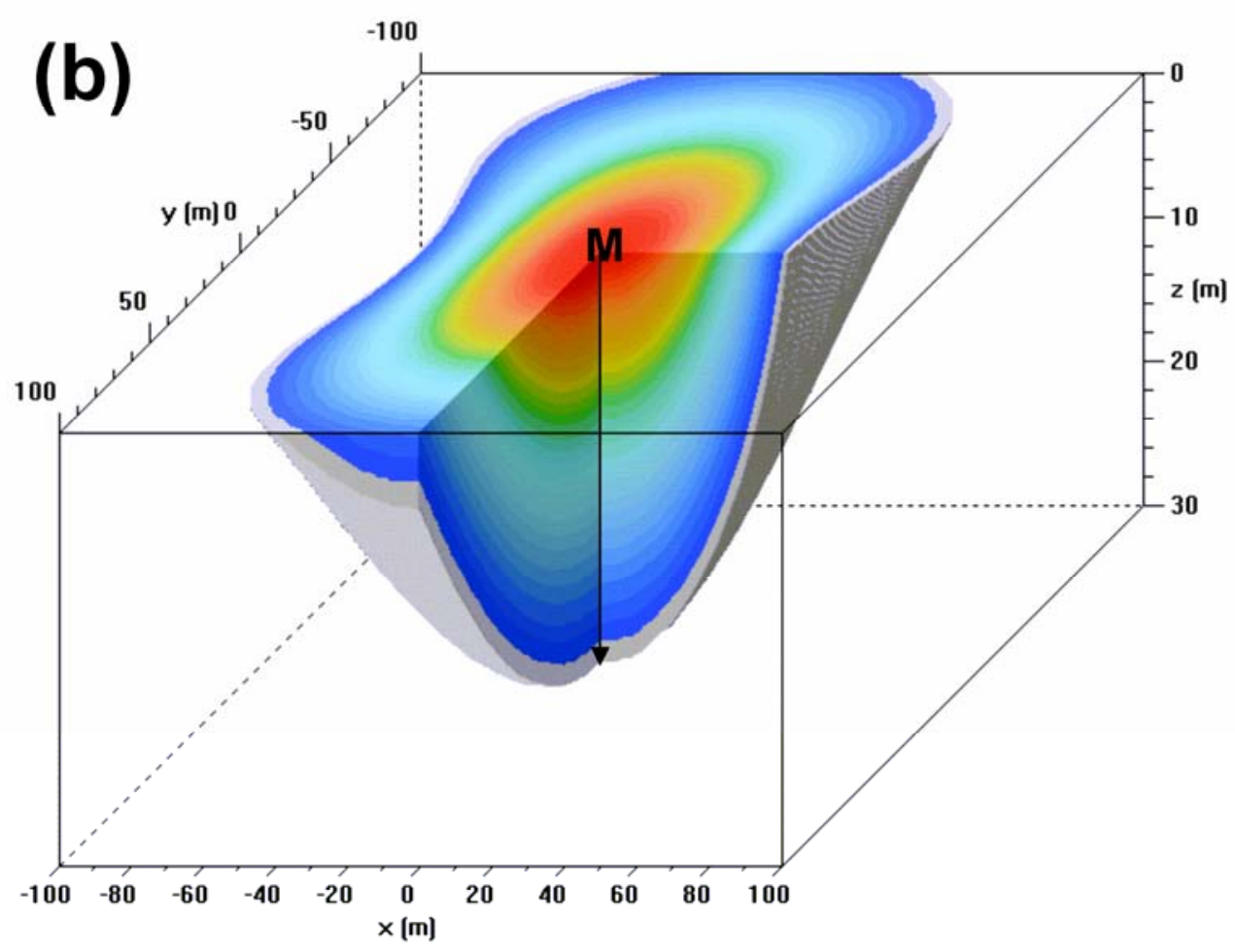

Figure 1 Three dimensional perspective views of induced electric field skin distance volumes (the contoured region) generated by magnetic dipoles at a frequency of 10 kHz, $90 \mathrm{~m}$ above a $20 \mathrm{mS} / \mathrm{m}$ half-space. (a) Vertical Magnetic Dipole (VMD) and (b) Horizontal Magnetic Dipole (HMD) polarised in the x-direction. The volume shown $(200 \times 200 \times 30 \mathrm{~m})$ has a vertical exaggeration of $\times 4$. The SE quadrant has been removed. 
a) HMD $P(x 10)$

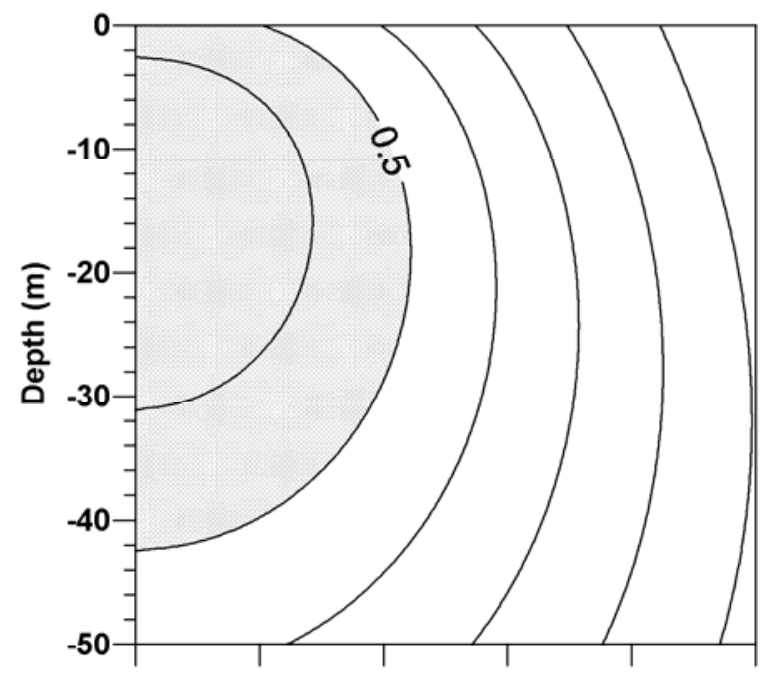

c) VMD $P(x 10)$

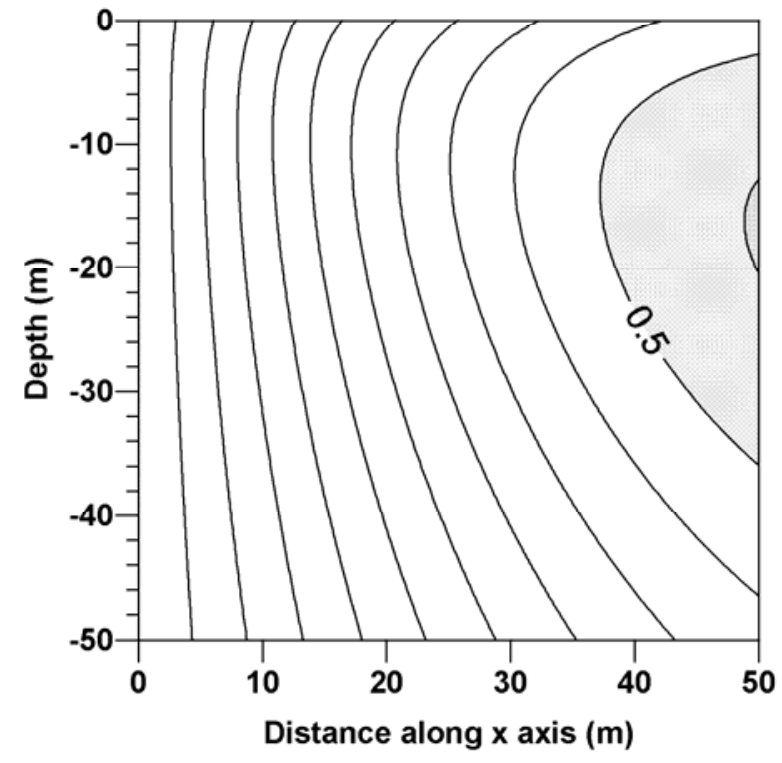

b) HMD Q

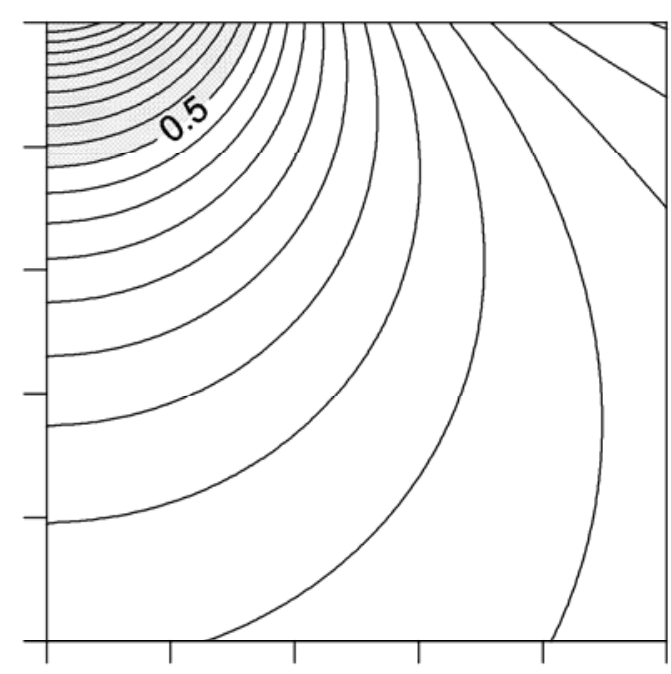

d) VMD Q

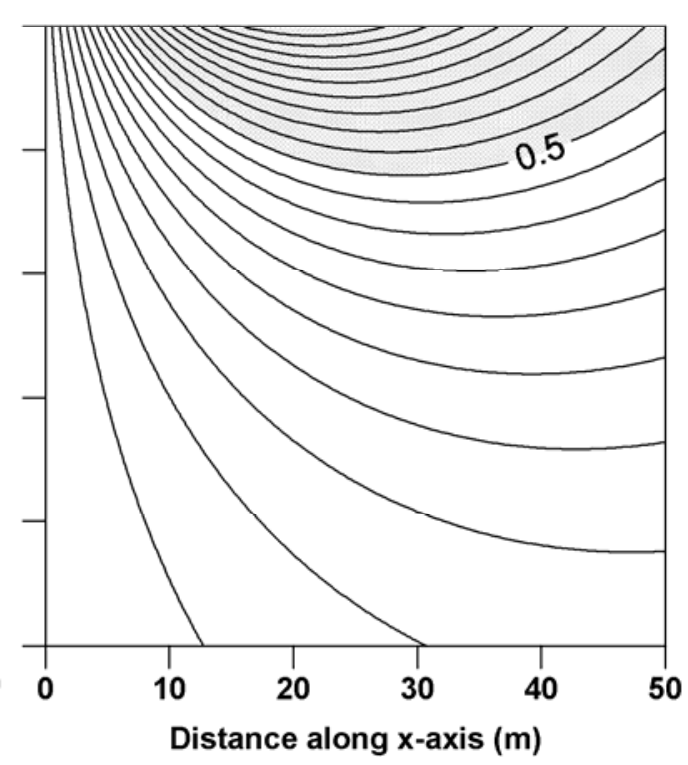

Figure 2 Cross sections, in the $\mathrm{x}-\mathrm{z}$ plane, of the induced electric field components $\mathrm{P}$ (in-phase) and Q (in-quadrature) due to (a) an Horizontal Magnetic Dipole (HMD) source polarised in the $\mathrm{x}$ direction, and (b) a Vertical Magnetic Dipole (VMD) source. In both cases the half space conductivity is $10 \mathrm{mS} / \mathrm{m}$, the frequency is $3 \mathrm{kHz}$ and the source is $30 \mathrm{~m}$ above the origin. The distributions have been normalised to a maximum value of unity and the in-phase values are shown multiplied by 10 . The maximum value is unity and the contour interval is 0.05 throughout. The contour interval is linear. 


\section{a) HMD}
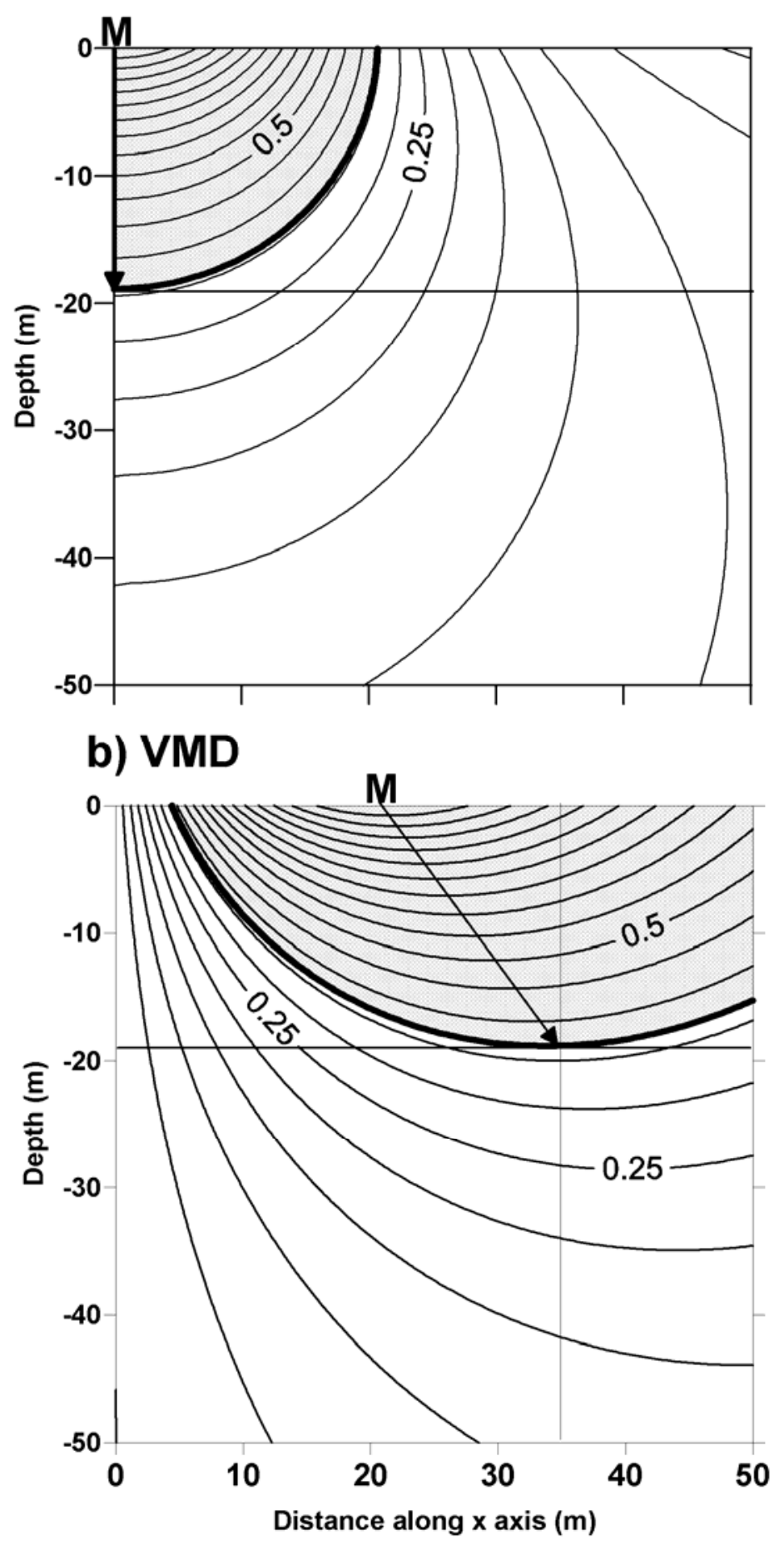

Figure 3 Cross sections, in the $x-z$ plane, of the modulus of the induced electric field due to a Horizontal Magnetic Dipole (HMD) source polarised in the $\mathrm{x}$ direction, and (b) a Vertical Magnetic Dipole (VMD) source. In both cases the half space conductivity is $10 \mathrm{mS} / \mathrm{m}$, the frequency is $3 \mathrm{kHz}$ and the source is $30 \mathrm{~m}$ above the origin. The distributions have been normalised to a maximum value of unity in each case and the contour interval is 0.05 . The heavy line contour (a value of 0.37 ) denotes a skin distance of $1 / \mathrm{e}$ from the maximum value of unity at the surface. 

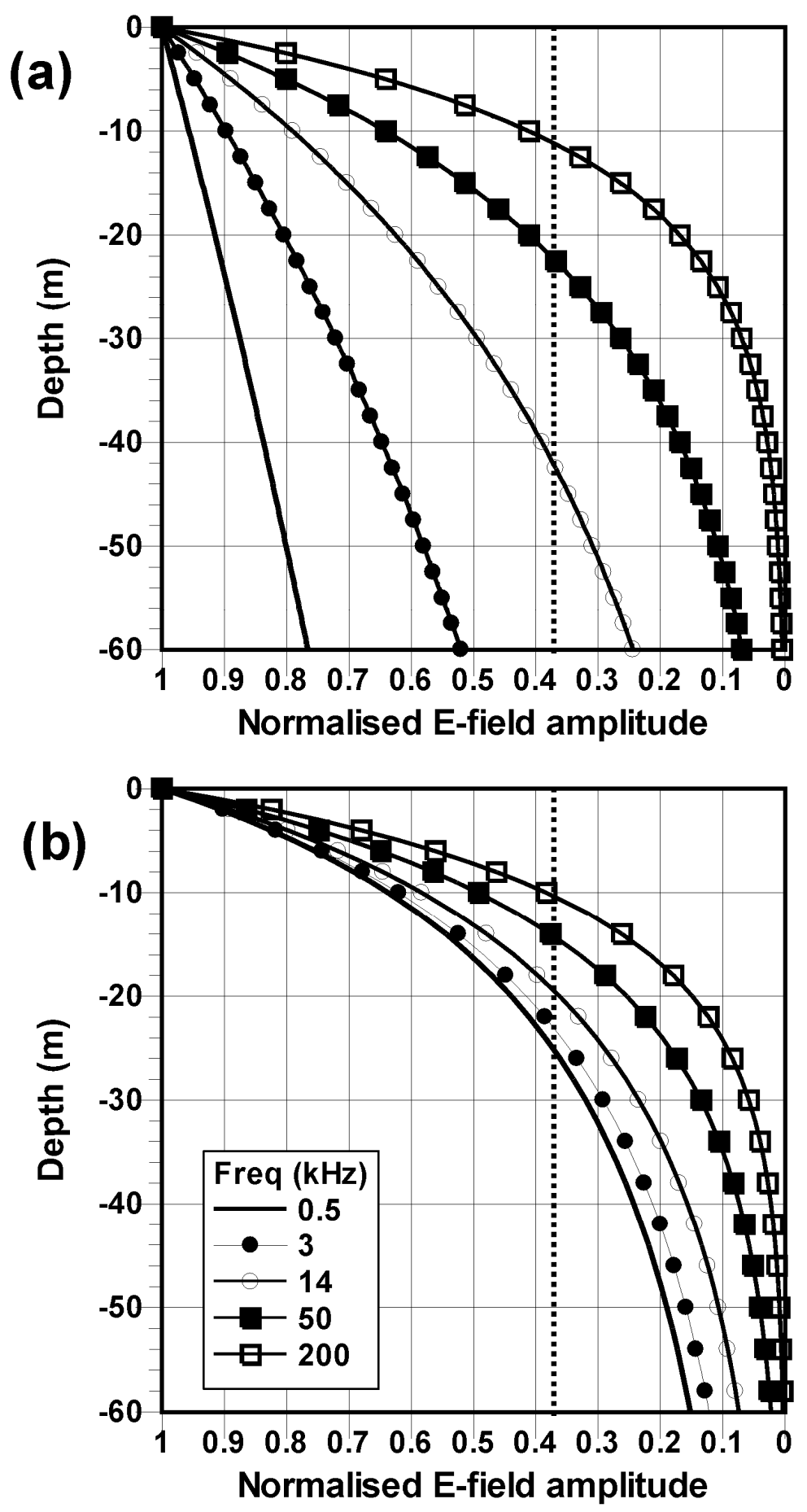

Figure 4 Comparison of the attenuation of induced electric fields, normalised to a value of unity at the surface, in the case of (a) a plane wave source and (b) a Vertical Magnetic Dipole (VMD) source, located $40 \mathrm{~m}$ above the half space. The half space conductivity is $10 \mathrm{mS} / \mathrm{m}$. Results for 5 frequencies are shown. The vertical dashed line denotes a value of $1 / \mathrm{e}(0.37)$ i.e. the skin depth. 


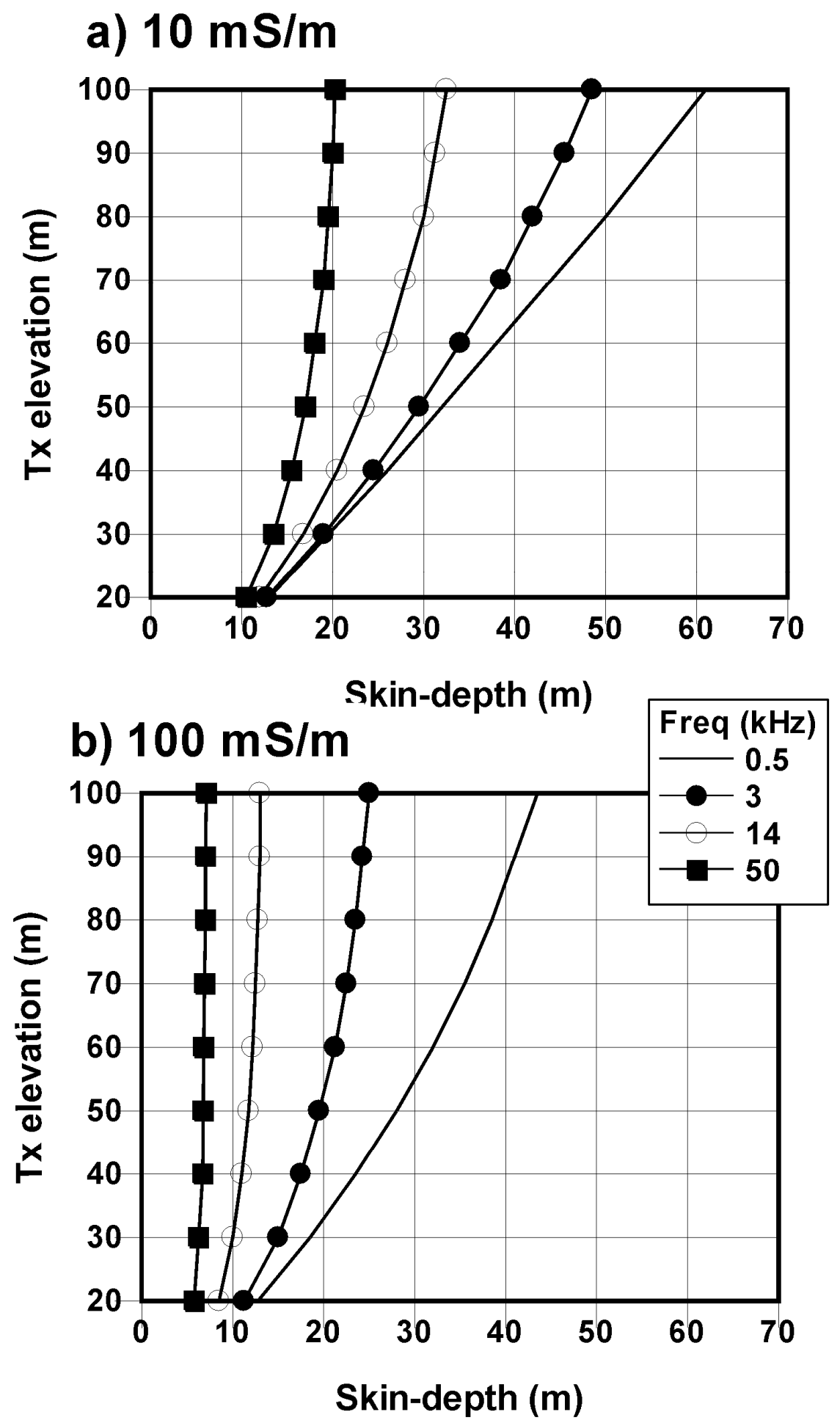

Figure 5 Variation of dipolar skin depth with source elevation and frequency for two half spaces (a) $10 \mathrm{mS} / \mathrm{m}$ and (b) $100 \mathrm{mS} / \mathrm{m}$. Results for 4 frequencies are shown as individual curves in each case. 
a) $500 \mathrm{~Hz}$

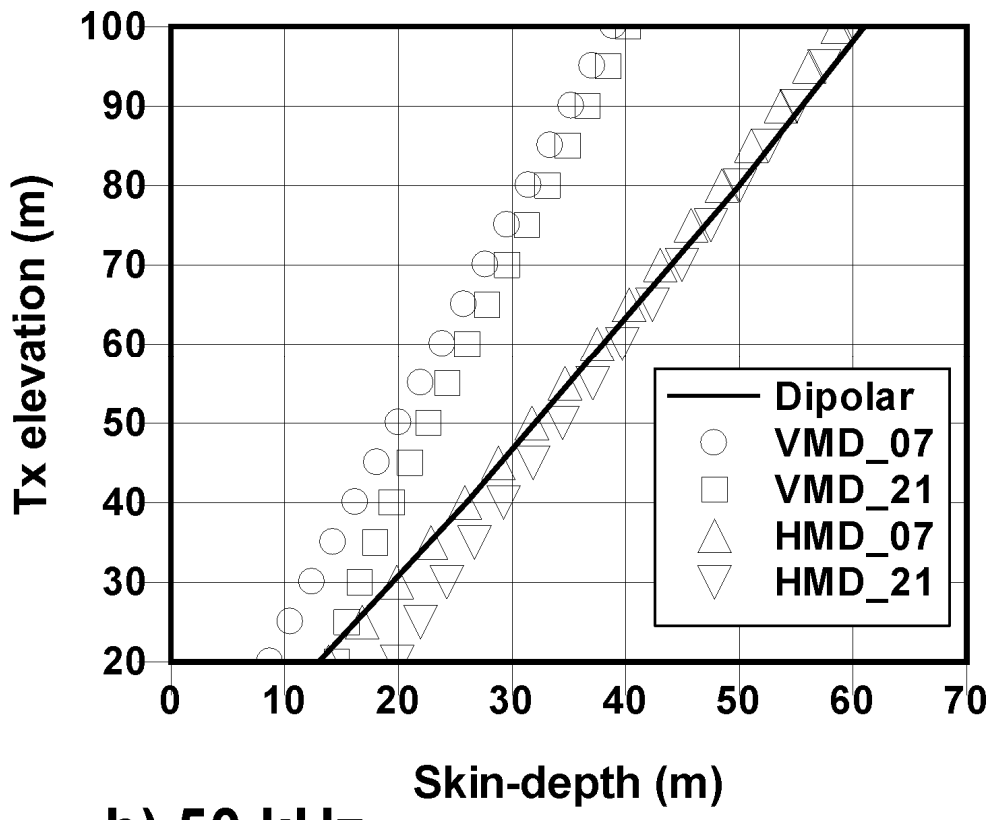

b) $50 \mathrm{kHz}$

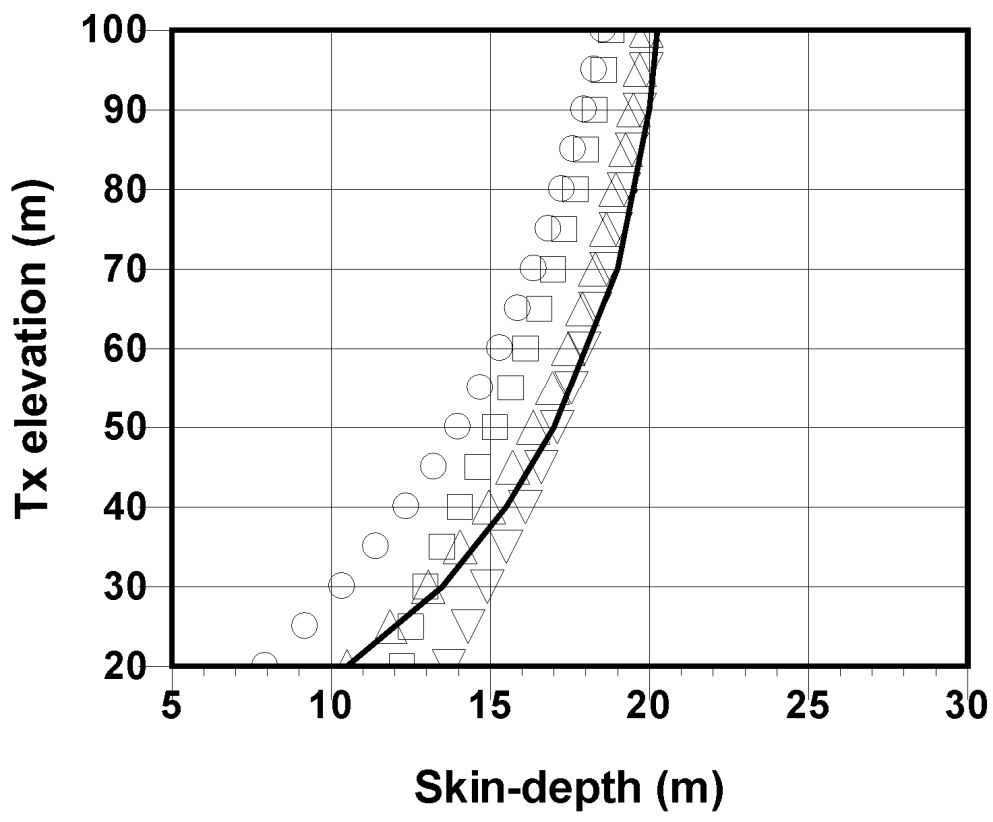

Figure 6 Comparison of dipolar skin depths (solid lines) with local source skin depths (symbols) for a half space of conductivity $10 \mathrm{mS} / \mathrm{m}$ and for frequencies of (a) $500 \mathrm{~Hz}$ and (b) $50 \mathrm{kHz}$. Local source skin depths are shown for Vertical Magnetic Dipoles (VMD) and for Horizontal Magnetic Dipoles (HMD) with transmitter-receiver offsets of $7 \mathrm{~m}$ (bird) and $21 \mathrm{~m}$ (fixed wing). 


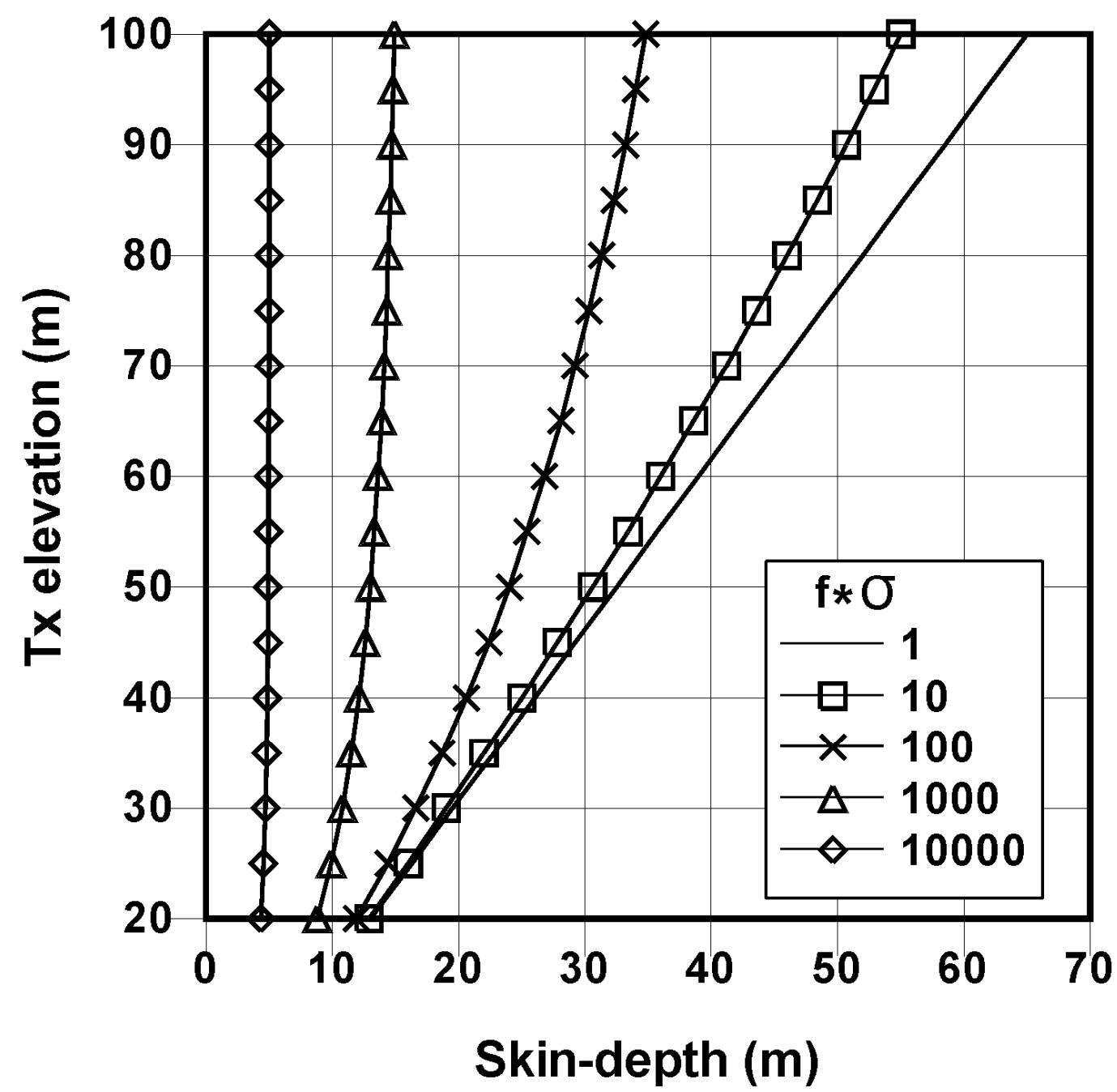

Figure 7 Variation of dipolar skin depth with elevation as a function of the frequencyconductivity product $\left(f^{*} \Phi\right)$. Five curves for the conductivity-frequency-conductivity product ranging from 1 to $10000 \mathrm{~Hz}$. S/m are shown. 


\section{a) $100 \mathrm{mS} / \mathrm{m}$}

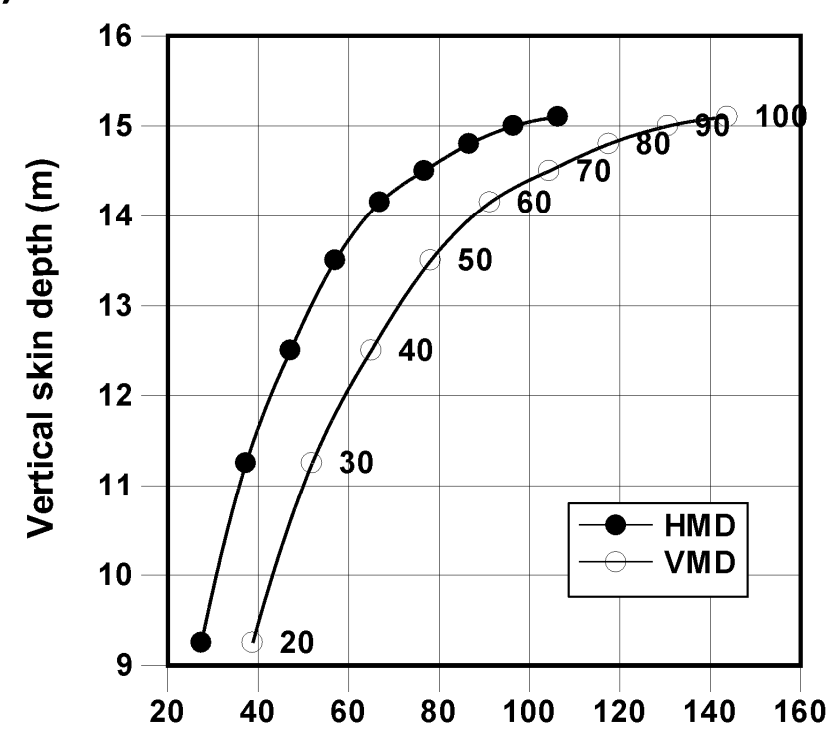

b) $1 \mathrm{mS} / \mathrm{m}$

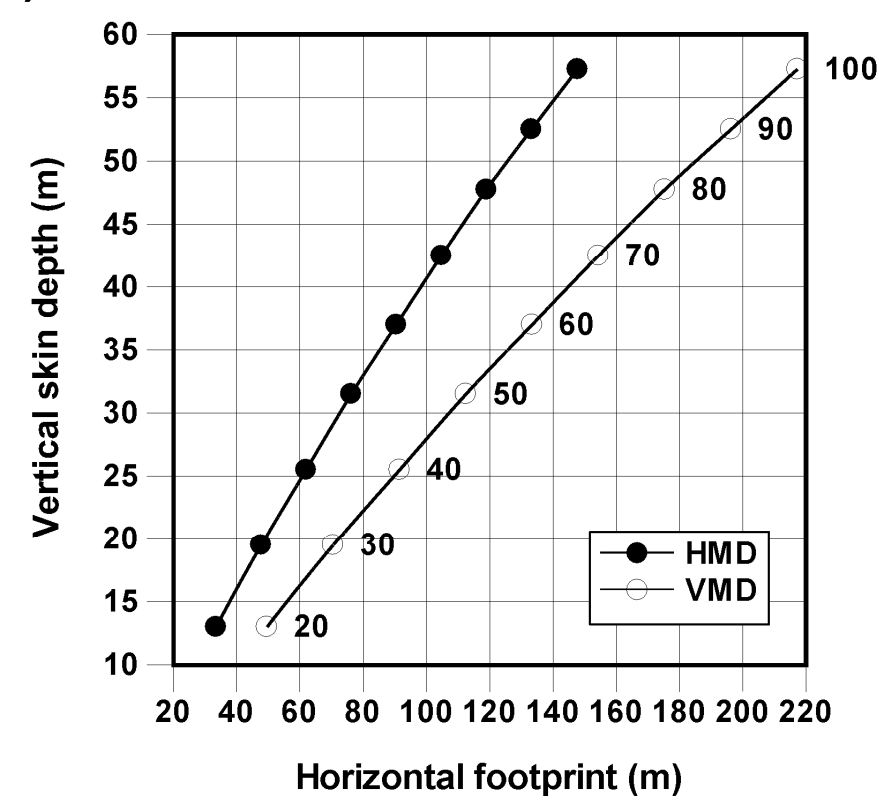

Figure 8 Variation of dipolar vertical skin depth with horizontal skin distance parameters (footprints) for VMD and HMD sources at elevations ranging from 20 to $100 \mathrm{~m}$. The numbers to the right of each symbol denote source elevation. The frequency used is $10 \mathrm{kHz}$. (a) Results for a half space conductivity of $100 \mathrm{mS} / \mathrm{m}$ and (b) Results for a half space conductivity of $1 \mathrm{mS} / \mathrm{m}$. 